\title{
La politique minière du gouvernement d'Evo Morales : entre mythes et pragmatisme politique
}

The mining policy of the Evo Morales government: Myths and political pragmatism

La política minera del gobierno de Evo Morales: entre mitos y pragmatismo político

\section{Claude Le Gouill}

\section{OpenEdition}

Journals

Édition électronique

URL : https://journals.openedition.org/ideas/1695

DOI : $10.4000 /$ ideas. 1695

ISSN : $1950-5701$

\section{Éditeur}

Institut des Amériques

\section{Référence électronique}

Claude Le Gouill, «La politique minière du gouvernement d'Evo Morales : entre mythes et

pragmatisme politique », IdeAs [En ligne], 8 | 2016, mis en ligne le 16 décembre 2016, consulté le 18 octobre 2022. URL : http://journals.openedition.org/ideas/1695; DOI : https://doi.org/10.4000/ideas. 1695

Ce document a été généré automatiquement le 18 octobre 2022

\section{(c) (†) $\odot$}

Creative Commons - Attribution - Pas d'Utilisation Commerciale - Pas de Modification 4.0 International - CC BY-NC-ND 4.0

https://creativecommons.org/licenses/by-nc-nd/4.0/ 


\section{La politique minière $\mathrm{du}$ gouvernement d'Evo Morales : entre mythes et pragmatisme politique}

The mining policy of the Evo Morales government: Myths and political pragmatism

La política minera del gobierno de Evo Morales: entre mitos y pragmatismo político

\section{Claude Le Gouill}

\section{Introduction}

1 Parmi les nombreux pays latino-américains à connaître un boom minier depuis les années 1990 (Warhust A. et Bridge G., 1997), la Bolivie est l'un des pays au passé minier important et autour duquel se sont créés les plus forts imaginaires. L'histoire de la Bolivie est en effet inséparable de celle de ses principaux gisements miniers, depuis la célèbre mine d'argent du Cerro Rico de Potosi à partir du XVIème siècle jusqu'aux gisements d'Oruro et de l'Altiplano qui firent la richesse des «barons de l'étain » au $\mathrm{XX}^{\mathrm{e}}$ siècle. Malgré cette richesse naturelle, le pays est considéré au début du troisième millénaire comme le pays le plus pauvre de l'Amérique du Sud et les zones minières comme les plus pauvres de Bolivie. L'expression « Ça vaut un Potosi » s'est ainsi peu à peu transformée en «syndrome de Potosi » : l'exploitation des ressources naturelles par une nation étrangère au détriment du développement de la population bolivienne (Perrier Bruslé L., 2007).

2 À côté de ce syndrome fortement ancré dans la conscience nationale coexiste l'imaginaire « eldoradiste » (Svampa M., 2011) de développement du pays à partir de sa seule base minière, porté par l'idéologie national-populaire. La première phase de ce projet intervient avec le gouvernement du Mouvement nationaliste révolutionnaire (MNR) (1952-1964) qui entreprend la nationalisation des mines avec la création de 
l'entreprise étatique Corporation minière de Bolivie (COMIBOL) et de la Centrale ouvrière bolivienne (COB), le puissant syndicat des travailleurs autour duquel se dessine l'imaginaire héroïque de la lutte révolutionnaire des mineurs contre les dictatures (1964-1982). La mise en place des politiques dites «néo-libérales» en 1985 marque cependant le démantèlement du modèle national-développementaliste avec la privatisation des grands secteurs miniers étatiques et par la même occasion la fin du mouvement ouvrier comme avant-garde de la contestation sociale.

3 Le deuxième projet «eldoradiste " intervient à la suite de la victoire du premier président indigène de Bolivie, Evo Morales, en 2005, et notamment de la reconnaissance de l'État plurinational à la suite de l'approbation par référendum en 2009 de la Nouvelle Constitution Politique de l'État (NCPE). Le projet politique porté par le gouvernement parvient à articuler les courants de pensée indianistes, marxistes et nationalistes révolutionnaires (Stefanoni P. et Do Alto H., 2006). Le point de convergence de ces différents courants se retrouve dans la reconquête des ressources naturelles en se basant sur le mythe commun «national-populaire» fortement ancré dans la population : l'affirmation de la Nation, un État redistributeur et l'articulation entre un leader charismatique et le peuple (Svampa M., 2010). Cette politique passe par un processus de nationalisation des ressources dites stratégiques - principalement du gaz ${ }^{1}$ - à des fins de redistribution ${ }^{2}$ et par un processus d'industrialisation des matières premières, qui, selon le vice-président bolivien Alvaro Garcia Linera, doit permettre au pays de sortir du modèle extractiviste en amassant des devises (Poupeau F., 2013). Ce nouveau modèle, qualifié de «néo-développementalisme » (Svampa M., 2011), se heurte cependant à l'imaginaire indigène mobilisé par le gouvernement et notamment au concept de "Vivir Bien ", institutionnalisé dans la NCPE et censé combiner certains principes éthiques - ou supposés comme tels - des cultures indigènes et les discours de certains courants intellectuels cherchant des alternatives au développement capitaliste (Vanhust J. et Beling A., 2013).

4 Après avoir suivi la voie de la libéralisation de son secteur énergétique comme 90 pays entre 1985 et 1995 (Yakovleva, 2005), la rhétorique indigène de la Bolivie doit en effet lui permettre de tracer - avec l'Equateur - une voie distincte au modèle "néolibéral » d'exploitation des ressources naturelles. Ce dernier marque en effet un retrait de l'État des secteurs stratégiques et doit permettre aux pays du Sud de s'adapter aux nouvelles conditions d'exploitation tout en maintenant les bénéfices sociaux et économiques de leurs économies minières (emplois, taxes, transferts technologiques...). Ainsi, si dans deux pays d'Amérique du Sud à avoir signé un accord de libre-échange avec les ÉtatsUnis - le Pérou et la Colombie - le discours contre la pauvreté est utilisé pour légitimer la politique extractiviste, à l'opposé, dans les pays « progressistes » comme la Bolivie, ce discours sur la pauvreté est remplacé par celui sur la souveraineté et la dignité face à l'investissement étranger (Gudynas E., 2009).

5 Si les contradictions entre la politique "néo-développementaliste » et le «Vivir Bien » sont aujourd'hui connues, la thématique minière offre un éclairage sur une autre tension : celle entre État et modes d'(auto-) organisation des bases sociales autour de la question des ressources naturelles ${ }^{3}$. Ce travail est l'occasion de dresser un état des lieux de la politique minière du gouvernement Morales ${ }^{4}$, au moment même où celle-ci connaît une crise sans précédent avec l'assassinat du vice-ministre de l'Intérieur par des membres de coopératives minières, lors d'un violent conflit en août 2016 faisant un bilan de cinq morts ${ }^{5}$. En effet, alors que dans les autres pays latino-américains les 
conflits miniers éclatent principalement en opposition aux projets d'exploitation, pour réclamer des droits, ou autour de questions environnementales (Bebbington A. et Humphreys Bebbington D., 2009), dans le cas bolivien ils s'orientent principalement vers la lutte entre organisations sociales pour le contrôle des ressources naturelles. Cette étude nous permettra de questionner la politique d'Evo Morales et d'analyser si elle répond à un programme planifié, ou si, au contraire, elle s'inscrit dans l'évolution du contexte minier national et international. La question du rôle central de l'État servira de fil directeur, avec dans un premier temps une présentation des différentes branches du secteur minier bolivien (section 1), puis une analyse de la politique minière gouvernementale (section 2), et enfin une réflexion autour du conflit d'août 2016 et ses impacts socio-politiques.

\section{Un secteur minier fragmenté}

6 Si le Movimiento al Socialismo (MAS) d'Evo Morales est parvenu à propager l'image d'un "gouvernement des mouvements sociaux », celle-ci apparaît cependant davantage comme un «mythe mobilisateur » (Zegada M. T., et al, 2011). En effet, le gouvernement doit faire face aux luttes corporatistes menées par les organisations sociales pour prendre le contrôle de ce "processus de changement». Le thème minier offre un bon exemple de ces luttes entre différents secteurs de la population. Lorsque Evo Morales remporte les élections en décembre 2005, ce secteur est divisé en trois branches: une branche étatique quasi inexistante depuis la privatisation des mines en 1985, une branche privée où les travailleurs sont affiliés - avec les travailleurs de la COMIBOL - à la Fédération syndicale des travailleurs mineurs de Bolivie (FSTMB) et la branche des coopératives réunies au sein de la Fédération nationale des coopératives minières (FENCOMIN).

7 Bien que hautement symbolique pour son rôle passé, l'activité minière a été détrônée par celle des hydrocarbures (gaz) comme moteur actuel de l'économie bolivienne. Elle représente en 2013 5,9\% du PIB, 19,9\% des exportations (3 083 millions de dollars d'exportation), 5,7\% des emplois, 7,7\% des impôts directs et $16,1 \%$ des redevances minières (comprenant les hydrocarbures et les minerais) (Sandi E., 2014). Selon les chiffres fournis par le gouvernement, le secteur privé assure en 2013 près de $80 \%$ de sa production et regroupe $6 \%$ des travailleurs. Bien qu'employant $90 \%$ de la maind'œuvre, estimée à près de 120000 travailleurs en 2013, les coopératives ne contribuent qu'à près de $17 \%$ de la production. Le secteur étatique assure quant à lui moins de $6 \%$ de la production (cf. tableau 1, p. 4). Une industrie minière «dualiste" s'est ainsi développée entre un secteur privé de grande productivité et un secteur de coopératives rudimentaires, et ce malgré une certaine réticence du premier à investir plus, du fait du manque de perspective politique stable du pays (Crabtree J. et Chaplin A., 2013).

Tableau 1 : Participation des différents secteurs à l'économie minière, 2005 et 2013

\begin{tabular}{|l|l|l|l|l|}
\hline & COMIBOL & Secteur privé & Coopératives & Total \\
\hline $\begin{array}{l}\text { Production 2005 } \\
\text { en tonnes métriques }\end{array}$ & & 182000 & 108000 & 290000 \\
$62,75 \%$ & $37,25 \%$ & $100 \%$ \\
\hline
\end{tabular}




\begin{tabular}{|c|c|c|c|c|}
\hline $\begin{array}{l}\text { Production } 2013 \\
\text { en tonnes métriques }\end{array}$ & $\begin{array}{l}27000 \\
3,68 \%\end{array}$ & $\begin{array}{l}581000 \\
79,37 \%\end{array}$ & $\begin{array}{l}124000 \\
16,94 \%\end{array}$ & $\begin{array}{l}732000 \\
100 \%\end{array}$ \\
\hline $\begin{array}{l}\text { Valeur } 2005 \\
\text { en millions de dollars }\end{array}$ & & $\begin{array}{l}347 \\
55 \%\end{array}$ & $\begin{array}{l}283 \\
45 \%\end{array}$ & $\begin{array}{l}630 \\
100 \%\end{array}$ \\
\hline $\begin{array}{l}\text { Valeur } 2013 \\
\text { en millions de dollars }\end{array}$ & $\begin{array}{l}292 \\
8,60 \%\end{array}$ & $\begin{array}{l}2112 \\
62,4 \%\end{array}$ & $\begin{array}{l}982 \\
29 \%\end{array}$ & $\begin{array}{l}3386 \\
100 \%\end{array}$ \\
\hline $\begin{array}{l}\text { Redevances } 2005 \\
\text { en millions de dollars }\end{array}$ & & $\begin{array}{l}10 \\
71,4 \%\end{array}$ & $\begin{array}{l}4 \\
28,6 \%\end{array}$ & $\begin{array}{l}14 \\
100 \%\end{array}$ \\
\hline $\begin{array}{l}\text { Redevances } 2013 \\
\text { en millions de dollars }\end{array}$ & $\begin{array}{l}13 \\
9,40 \%\end{array}$ & $\begin{array}{l}94 \\
68,1 \%\end{array}$ & $\begin{array}{l}31 \\
22,5 \%\end{array}$ & $\begin{array}{l}138 \\
100 \%\end{array}$ \\
\hline $\begin{array}{l}\text { Nombre de } \\
\text { travailleurs en } 2005\end{array}$ & $\begin{array}{l}117 \\
0,2 \%\end{array}$ & $\begin{array}{l}5450 \\
9,80 \%\end{array}$ & $\begin{array}{l}50150 \\
90 \%\end{array}$ & $\begin{array}{l}55717 \\
100 \%\end{array}$ \\
\hline $\begin{array}{l}\text { Nombre de } \\
\text { travailleurs en } 2013\end{array}$ & $\begin{array}{l}7902 \\
5,8 \%\end{array}$ & $\begin{array}{l}8110 \\
6 \%\end{array}$ & $\begin{array}{l}119340 \\
88,2 \%\end{array}$ & $\begin{array}{l}135352 \\
100 \%\end{array}$ \\
\hline
\end{tabular}

Source : Élaboration propre à partir des chiffres du rapport de gestion 2013 de l'État Plurinational de Bolivie

\section{Un secteur étatique en croissance}

8 Alors que la NCPE indique que toute activité économique doit renforcer la souveraineté du pays (NCPE, 2009), à travers notamment la nationalisation des ressources naturelles et leur industrialisation, la politique minière du gouvernement semble cependant moins orientée par une planification étatique de l'économie qu'influencée par la conjoncture internationale de la hausse des prix des matières premières. Le gouvernement déclare en 2007 l'ensemble du territoire national comme réserve fiscale (Oporto H., 2013). La COMIBOL est alors chargée de répertorier les gisements stratégiques qu'elle souhaite exploiter, mais les pressions des autres secteurs miniers (privés et coopératives) et le manque d'experts, depuis la fermeture de l'Institut de recherches sur les minéraux et métallurgie en 1985, la prive de toute capacité technique pour mettre à bien ce projet (entretien Hector Cordova ${ }^{6}$, La Paz, avril 2014).

9 Ce manque de ressources humaines, mais aussi les problèmes d'approvisionnement énergétique et de transport, ainsi que les pressions des entreprises étrangères (ibid.), compliquent également le processus d'industrialisation des minéraux ${ }^{7}$. Cette industrialisation permettrait au pays de sortir de sa condition de simple producteur de matières premières, et au gouvernement de légitimer l'ensemble de ses politiques «néo-développementalistes ».. En dehors de ces difficultés, la COMIBOL doit en plus gérer les conflits d'intérêt entre les secteurs privés et les coopératives minières qui se partagent - parfois sur un même territoire - les gisements les plus importants du pays (voir infra). Depuis l'élection d'Evo Morales, la COMIBOL a ainsi connu six présidents en huit ans, dont trois pour la seule année 2012 (Oporto H., 2013). 
10 Le retour du thème minier sur la scène nationale a entraîné la résurgence du mouvement ouvrier ${ }^{8}$, bien que celui-ci soit marqué par des divisions organisationnelles et idéologiques. Certains aspects nationalistes de la politique gouvernementale ont permis au secteur de la Fédération syndicale des travailleurs miniers de Bolivie (FSTMB) - qui réunit à la fois les travailleurs salariés du secteur étatique et du secteur privé - de retrouver un élan nationaliste révolutionnaire passé (Quiroga Trigo M. S., 2014), en réactivant un discours socialiste dans d'importantes mobilisations destinées à renforcer les droits de l'ensemble des travailleurs (augmentation des salaires, loi des retraites). D'autres demandes sont au contraire directement destinées au secteur minier salarié, en franche opposition au secteur des coopératives: demande de nationalisation de certaines aires de travail (Colquiri, Humalaya), généralisation de l'impôt au secteur des coopératives (ibid.). Les revendications socialistes ont également leurs limites, du fait que la FSTMB représente à la fois les syndicalistes du secteur public et du secteur privé. Elle s'oppose ainsi à cette "nationalisation » accompagée de la reprise du contrôle d'entreprises minières par l'État, des entreprises minières privées en affirmant que cela va contre les travailleurs, sous prétexte que la COMIBOL n'aurait pas les moyens de l'administrer.

\section{Un secteur privé privilégié mais prudent}

11 Lors de la victoire d'Evo Morales, les entreprises privées étaient assurément celles qui avaient le plus à craindre les évolutions de la politique gouvernementale. Les principales entreprises - Inti Raymi (Promisa), Sinchi Wayra (Glencore), Manquiri (Coeur d'Alene Mines Corporation), Empresa Minera Mallku Khota (South American Silver) - possèdent des droits d'exploitation avec la COMIBOL à travers des contrats de location ou de risques partagés. La mine de San Cristobal (de la transnationale japonaise Sumitomo) était la seule à bénéficier d'une concession privée. Avec un investissement total de 2 milliards de dollars et 1500 travailleurs, il s'agissait de la mine la plus importante de Bolivie avec une production journalière de 40000 tonnes d'argent concentré (CEDLA, 2014a). Plus de 60\% des redevances minières proviennent de 5 entreprises privées, dont $29 \%$ pour la seule entreprise San Cristobal dans le département de Potosi (ce qui représente 70\% des redevances totales du département).

Depuis la privatisation des mines d'État en 1985, ces entreprises avaient bénéficié de nombreux avantages fiscaux et légaux. La Loi Environnementale 1333 de 1992 établissant l'impératif de Licence Environnementale, dont les études des impacts environnementaux étaient réalisées par l'opérateur minier lui-même et validées par une consultation extérieure spécialisée sans l'intervention de l'État, avait permis la création d'un marché de services entre consultants et opérateurs au détriment de toute prise en compte sérieuse des impacts environnementaux. Ces entreprises ont également su s'adapter au changement politique en cours en Bolivie. Tout d'abord en changeant de nom, afin d'adopter des noms quechua et liés à la nature comme les entreprises Inti Raymi («fête du soleil » en quechua) ou Sinchi Wayra («vent fort » en quechua), ou en mettant en place une politique de Responsabilité sociale entrepreneuriale (RSE) (Rodriguez-Carmora et al., 2013), conformément aux règles de bonne conduite promues par l'industrie minière au niveau international (Yakovleva N., 2005). 
13 Malgré les craintes des entreprises minières envers le discours nationaliste et souverainiste du gouvernement d'Evo Morales, le secteur privé n'a connu qu'une modification partielle du régime tributaire. Si le montant des redevances n'a pas été relevé, bien qu'il ait été établi lorsque le prix des matières premières était encore faible, le gouvernement a néanmoins ajouté une taxe de $25 \%$ sur les services ainsi qu'une taxe extraordinaire de $12,5 \%$ en cas de forte augmentation des prix (voir tableau 2, p.8). Mais alors que dans le secteur des hydrocarbures le taux d'imposition est de $50 \%$, dans le secteur minier il est passé d'un taux de $3 \%$ avant l'élection d'Evo Morales à seulement $8 \%$ aujourd'hui.

D'après le témoignage d'un technicien d'une entreprise qui a désiré conserver l'anonymat (entretien en avril 2014, La Paz), la plus grande difficulté pour le secteur privé a ainsi été le « vide juridique » qui a fait suite à la mise en place de la NCPE en 2009, et ce jusqu'à la Loi minière de 2014. Ce vide explique ainsi la baisse de l'investissement privé de 49,2\% entre 2006-2009 et 2010-2013 (Sandi, 2014). Pourtant, toujours d'après cette source, son entreprise n'a jamais douté de la possibilité de trouver des compromis avec le gouvernement. Comme il l'affirme, "que les règles soient bonnes ou mauvaises, il y a un terrain où jouer ». Plusieurs lois ont d'ailleurs renforcé la confiance des entreprises, notamment la loi contre les invasions de gisements. Quant à l'approbation de la Loi de la Terre-Mère, sensée renforcer la précédente loi de 1992, ce même technicien indique: "De la loi de l'environnement à la loi de la Terre-Mère, le nom change, mais la loi et les règlements comme tels n'ont rien changé, ça reste les mêmes, même s'il y a un cadre général de protection pour le changement climatique. " (entretien en avril 2014, La Paz).

\section{Un secteur « coopérativiste » en forte augmentation}

15 La Fédération nationale des coopératives minières (FENCOMIN) est fondée dans les années 1960 dans les petites mines du pays. À partir de la privatisation de 1985, la coopérative devient l'unique force légale pour exploiter les mines face à la disparition de la COMIBOL et l'absence de travail dans les entreprises privées. Ce secteur connaît une forte croissance à partir de la hausse des prix des matières premières (voir tableau 1, p.5). Alors que le nombre de coopératives était de 690 en 1995, il passe à 1642 en 2014. Les aires opérées par les coopératives ont quant à elles augmenté de 700\% depuis 2006 (Quiroga Trigo M.S., 2014). La productivité du système coopératif reste cependant extrêmement faible du fait du peu d'investissement et du manque de mécanisation. De plus, seulement $16 \%$ des travailleurs des coopératives bénéficieraient de la sécurité sociale et d'après l'UNESCO, il y aurait eu 120 morts dans les galeries du Cerro Rico de Potosi rien que pour l'année 2012 (Francescone K. et Diaz V., 2013).

16 En Bolivie, la coopérative est reconnue par la Loi générale des sociétés coopératives de 1958 qui la définit par l'égalité des droits et les obligations des partenaires en termes de gestion démocratique, de contrôle social, de finalité sociale et de limitation d'intérêt du capital. C'est au nom de ce caractère "social» que les coopératives minières boliviennes bénéficient d'avantages en étant exemptées de certains impôts (tableau 2, p.6) et des contraintes environnementales. Pourtant, de nombreuses études ont montré que la structure même des coopératives minières est très inégalitaire en Bolivie, avec en haut de la pyramide une élite dirigeante administrative et en bas les travailleurs de la mine, les peones, sans statut juridique ni droit de se syndicaliser, souvent engagés par 
les socios de la coopérative. Dans les galeries, la logique unitaire syndicale et l'esprit de lutte de la $\mathrm{COB}$ ont ainsi laissé place à un usufruit du travail individuel et à une conduite plus pragmatique orientée vers la réussite d'intérêts corporatistes immédiats en accord avec le modèle extractiviste (Michard J., 2008 ; Francescone K. et Diaz V., 2013 ; Quiroga Trigo M.S., 2014). Sur les près de 1700 coopératives minières actuelles du pays, $30 \%$ opéreraient de manière illégale selon le vice ministère des Coopératives, parmi lesquelles $65 \%$ sont spécialisées dans l'or (dont $91 \%$ dans le département de La Paz) (Poveda A., 2014).

17 Ces coopératives sont ainsi considérées par les mineurs syndicalistes des secteurs privé et public comme la "nouvelle bourgeoisie » de l'industrie minière (Crabtree J. et Chaplin A., 2013). À la différence de la FSTMB, la FENCOMIN se caractérise par un discours uni de défense totale de ses intérêts corporatifs. De par le nombre de leurs membres, les coopératives jouent un rôle politique majeur. En 2005, Evo Morales aurait ainsi proposé aux coopératives de Huanuni des aires de travail en échange de leur appui dans les urnes. Cet accord "pas très intelligent mais attractif électoralement " engendre par la suite de violents affrontements avec les secteurs syndicalistes (voir infra) (Portugal P., 2012, p.191-192). Sous la pression de la FENCOMIN, le gouvernement proclame plusieurs lois en faveur des coopératives. En 2011 la Loi 186 instaure le régime à taux zéro de l'IVA pour la commercialisation de leur production. À la suite de la «Première rencontre plurinationale pour approfondir le changement ", en 2012, la COMIBOL entreprend une « libération » d'une partie de ses réserves fiscales, établies en 2007, au bénéfice des coopératives (Quiroga Trigo M.S., 2014 ; Arze Alegria A., 2014).

Malgré les tensions entre ces différents secteurs, un point permet leur unité : la menace que représente pour eux les secteurs indigènes. Tant les coopératives que les syndicats se sont en effet sentis dépassés et en manque de ressources face aux demandes de "décolonisation » des secteurs indigènes, même si la FSTMB a montré une certaine sensibilité en émettant en urgence (face à l'ampleur nationale de la mobilisation) une résolution en "Appui et solidarité avec les "camarades" du TIPNIS", mobilisés en défense du Territoire indien et parc national Isiboro - Sécure (TIPNIS) contre un projet de construction de route (Quiroga Trigo, 2014). À côté de cela, la FSTMB demande la destitution de fonctionnaires du Ministère de l'Environnement et de la Terre-Mère d'Oruro, jugés trop proches d'ONG environnementales (Quiroga Trigo, 2014) et apporte son soutien aux secteurs privés contre les prises d'assaut de certaines concessions par les secteurs indigènes. En 2013 est approuvée la Loi 477 contre les invasions et trafics de terres, condamnant les assauts indigènes sur les aires de travail minières 9 . Un autre point d'accord entre secteurs miniers sur cette thématique indigène a été celui des " entreprises communautaires ». La FSTMB rejette ces projets qu'elle considère comme des plans d'expropriation de propriétés et d'entreprises au seul bénéfice des peuples indigènes. De con côté, la FENCOMIN juge ces projets communautaires comme une véritable concurrence au système de coopératives. Elle affirme à plusieurs reprises que ce mode de gouvernance minière n'a aucune existence légale mais que les communautés pouvaient créer leurs propres coopératives paysannes-indigènes au sein de la FENCOMIN, en précisant que $30 \%$ des coopératives fonctionneraient selon ce modèle. 
Tableau 2 : Les impôts par secteur minier

\begin{tabular}{|c|c|}
\hline Principaux Impôts & Quels secteurs financent \\
\hline $\begin{array}{l}\text { Redevances minières: entre } 1 \% \text { et } 7 \% \text { de la valeur de production } \\
\text { (selon le minerai et les cotisations sur le marché) }\end{array}$ & $\begin{array}{l}\text { - COMIBOL } \\
\text { - Entreprises privées } \\
\text { - Coopératives }\end{array}$ \\
\hline $\begin{array}{l}\text { Impôt sur les Bénéfices des Entreprises : } 25 \% \text { de la valeur de la } \\
\text { production }\end{array}$ & $\begin{array}{l}\text { - COMIBOL } \\
\text { - Entreprises privées }\end{array}$ \\
\hline $\begin{array}{l}\text { Impôt additionnel à l'impôt sur les Bénéfices des Entreprises (lorsque } \\
\text { le prix des minéraux est fort) : } 12,5 \% \text { de la valeur de la production }\end{array}$ & $\begin{array}{l}\text { - COMIBOL } \\
\text { - Entreprises privées }\end{array}$ \\
\hline \multicolumn{2}{|l|}{ Taxes supplémentaires } \\
\hline Taxe de location à la COMIBOL & $\begin{array}{l}\text { Coopératives: } 1 \% \text { de la } \\
\text { valeur de production }\end{array}$ \\
\hline $\begin{array}{l}\text { Impôt sur la Valeur Ajoutée IVA (sur les ventes internes de } \\
\text { minéraux) }\end{array}$ & Entreprises privées \\
\hline Régime Complémentaire sur l'IVA (sur les excédents de perceptions) & Entreprises privées \\
\hline Impôt sur les Transactions (sur les ventes internes de minéraux) & Entreprises privées \\
\hline $\begin{array}{l}\text { Impôt Consolidé Coopératif Minier (en projet au sein du nouveau } \\
\text { code minier) }\end{array}$ & $\begin{array}{l}\text { Coopératives: } 1 \% \text { de la } \\
\text { valeur brute des ventes }\end{array}$ \\
\hline
\end{tabular}

Source : Élaboration par l'auteur à partir de différents documents de presse et d'entretiens réalisés en avril 2014 à La Paz et Cochabamba.

\section{La politique minière du gouvernement d'Evo Morales}

La politique minière du gouvernement Morales se déploie à la fois dans une période de hausse du prix des matières premières (de 2006 à 2011) et de transition entre l'ancien Code minier «néo-libéral » de 1997, l'approbation de la NCPE en 2009 et la Loi minière de 2014 censée accorder ce secteur aux normes de l'État plurinational. Cette phase de transition n'a pas été sans effet sur le secteur minier. En effet, entre les périodes de 2006-2009 et 2010-2013, soit avant et après l'approbation de la NCPE, les investissements à destination du secteur minier diminuent de $29,8 \%$, du fait principalement du vide juridique entourant l'activité minière et de l'augmentation des conflits sociaux autour de cette activité (Sandi E., 2014). La situation est cependant différente entre le secteur public qui connaît une forte augmentation à la suite des projets d'industrialisation $(186,5 \%)$ et l'investissement privé en forte en baisse $(49,2 \%)$. Afin de stimuler les investissements et d'ouvrir de nouvelles aires de travail, le gouvernement approuve en 2013 la Loi 403 de Réversion minière permettant de reverser les concessions peu enclines à l'investissement et n'accomplissant pas de 
"fonction économique et sociale ". Grâce à cette mesure, il espérait reverser $70 \%$ de concessions minières privées mais il n'a jamais atteint cet objectif (Poveda A., 2014).

\section{Un processus de nationalisation « accidentel »}

20 Si le processus de nationalisation touche en priorité les hydrocarbures (gaz), plusieurs mines sont cependant nationalisées à partir de 2006. Il s'agit néanmoins davantage d'une nationalisation politique que stratégique, destinée à mettre un terme aux luttes de pouvoir entre secteurs miniers pour le contrôle des gisements. La mine de Huanuni, dans le département d'Oruro, est nationalisée à la suite de violents heurts opposant les 800 salariés de la COMIBOL aux 4000 « coopérativistes ». A Colquiri, le conflit oppose les 400 salariés de l'entreprise Sinchi Wayra (de la transnationale suisse Glencore) et 1200 «coopérativistes » qui voulaient expulser l'entreprise étrangère au nom de la "souveraineté nationale ». Après de multiples affrontements faisant plusieurs morts, de nombreux blessés et occasionnant même des prises d'otage, les $45 \%$ de la mine appartenant à l'entreprise Sinchi Wayra sont nationalisés en 2011, alors qu'une partie de la mine - la partie dénommée Rosario, la plus riche - est cédée aux « coopérativistes». Cette nationalisation «partielle» de Colquiri n'a cependant pas atténué les tensions qui se reproduisent en 2012 et 2013. Ces nationalisations ont fortement augmenté le nombre de travailleurs au sein de la $\mathrm{COMIBOL}^{10}$, mais n'ont pas permis d'améliorer la productivité des gisements par manque d'investissement, ce qui amena Evo Morales à déclarer au Parlement : «si la nationalisation est pour produire moins, alors il ne faut pas nationaliser » (Oporto, 2013).

21 Le gouvernement d'Evo Morales impulse en 2012 une troisième nationalisation, celle du gisement de Mallku Khota, dans la région du Nord Potosi, contrôlé par l'entreprise « junior» canadienne South American Silver à travers l'Empresa Minera Mallku Khota. A la différence de Huanuni et de Colquiri, cette nationalisation se base non pas sur un conflit entre mineurs, mais sur un conflit opposant l'entreprise aux communautés paysannes-indigènes désirant constituer leur propre entreprise communautaire (Le Gouill C., 2016). La mine de Mallku Khota est finalement nationalisée le 1er août 2012 après des mois de conflits faisant un mort et des dizaines de blessés entre opposants et partisans de l'entreprise, tous issus d'un monde rural fortement divisé.

Comme l'indique Jorge Companini du Centre de documentation et d'information de Bolivie (CEDIB) (entretien avril 2014, Cochabamba), ces nationalisations sont plus le fruit d' "accidents ", liés à des pressions sociales, que d'une véritable stratégie économique sur le long terme. Pour Henry Oporto (2013), reprenant un concept de Max Weber, cette nationalisation s'apparente ainsi à un «capitalisme politique»: la nationalisation se fait sur des critères politiques et non pas économiques, dont l'évènement le plus récent est la nationalisation accompagnant les violences d'août 2016 (voir infra).

\section{Une nouvelle Loi minière controversée}

Sur le thème minier, la NCPE avait défini de nouvelles limites à la superficie des concessions minières et introduit leurs obligations de "fonctions économiques et sociales ». Elle avait surtout remplacé ces concessions par des contrats imprescriptibles, intransférables et intransmissibles par succession héréditaire (à 
l'opposé des concessions héritées de 1997). La nouvelle Loi minière, approuvée par le parlement en mai 2014, avait pour objectif de les renforcer. Cette loi est élaborée par les organisations minières et les cabinets ministériels sans la présence des organisations paysannes-indigènes. Le projet suscite de nombreuses critiques, tant de la part des ONG environnementalistes que de nombreuses organisations sociales pour son caractère anticonstitutionnel, anti-étatique et anti-indigène (CEDIB, 2014).

De la même manière que la NCPE, la Loi minière fait du peuple bolivien le propriétaire des ressources naturelles, mais leur administration est confiée à l'État comme représentant du peuple (Poveda A., 2014). La Loi minière, dans son article 17, reconnaît la «fonction économique et sociale » de l'activité minière. Celle-ci renvoie cependant uniquement à l'exercice de l'activité et au paiement de la patente minière, sans prendre en compte l'intérêt étatique, les normes environnementales ou un quelconque programme de développement local (CEDLA, 2014b).

25 La principale modification par rapport au code minier précédent concerne les modalités de concession qui deviennent des contrats et licences. Le «contrat d'association » octroie un partage des bénéfices de $45 \%$ pour l'entreprise et de $55 \%$ pour l'État, excepté pour la mine de San Cristobal qui reste une concession privée. Les contrats signés avant la mise en place de cette nouvelle loi sont cependant maintenus. Pour les nouveaux, il est nécessaire d'obtenir un contrat administratif minier de la part de l'Autorité juridictionnelle administrative minière (AJAM). L'acteur minier doit aussi être reconnu comme tel, et prouver un plan de travail et des outils de suivi et de contrôle. Le contrat administratif a une durée de 30 ans, mais pourra être suspendu et redonné à l'État si le paiement de la patente minière ne se fait pas, en cas de suspension des activités ou de non respect du plan de travail. Ce droit de l'État de maintenir ou de suspendre le contrat peut cependant engendrer des conflits sociaux et des pressions de groupes extérieurs pour influencer ses décisions (Sandi E., 2014).

Plusieurs points de la loi ont été jugés anticonstitutionnels par les organisations indigènes, du fait que les peuples autochtones ne soient pas reconnus comme « sujets de droits miniers", les privant ainsi du droit de création $d^{\prime}$ ' entreprises communautaires " gérées directement par les communautés. La loi renforce également un peu plus la criminalisation de la protestation (article 99-II), offre aux entreprises un accès illimité aux ressources hydriques et forestières, ainsi qu'un accès aux terres des communautés (article 107, 108), tout en n'offrant aucune garantie pour celles-ci quant à leur participation aux bénéfices de l'exploitation, cette participation devant être négociée avec les départements. L'un des principaux sujets de discorde est l'adoption limitée du droit international de «consultation préalable " ${ }^{11}$, en termes de définition restreinte des peuples indigènes (article 209), de son encadrement par l'AJAM, d'absence de droit de veto et de la limitation de la consultation aux nouveaux contrats miniers signés après l'application de cette loi (article 207) (Madrid E., 2014; Sandi E., 2014). Evo Morales avait, sur ce sujet de la " consultation préalable ", qualifié celle-ci de " chantage » (Rodriguez-Carmora A. et al., 2013) ou d' " obstacle au développement de la Bolivie » (Madrid E., 2014).

27 Les coopératives de leur côté bénéficient avec cette nouvelle loi des mêmes droits que les entreprises privées, et même certains avantages supplémentaires, comme les aires de location de la COMIBOL qui peuvent se transformer en contrats administratifs de durée indéfinie en les libérant du paiement de la location (article 63). Le débat le plus important concerne les articles autorisant les coopératives à passer des accords avec les 
entreprises multinationales, ce qui permet à ces dernières de conquérir de nouvelles aires de travail tout en bénéficiant du caractère "social» des coopératives pour être exemptées de certains impôts et du contrôle environnemental. Ces accords étaient déjà connus et inscrits dans la NCPE (article 351) mais leur institutionnalisation dans la Loi minière (article 151) a engendré de vives critiques - même de la part de membres du gouvernement - à tel point qu'ils ont été supprimés avant l'approbation de la loi au parlement. S'il n'est plus permis aux coopératives de passer des accords avec des transnationales, l'article 130 établit cependant que les contrats passés avant la loi sont maintenus, alors qu'ils étaient illégaux.

Malgré le retrait de l'article 151, les « coopérativistes" sont ainsi une nouvelle fois les grands gagnants de la politique minière. De par leur nombre et leur poids électoral, ils parviennent à influencer le gouvernement alors même que les coopératives vont, dans les faits, à l'encontre du projet souverainiste et redistributeur du MAS. Non seulement leurs redevances ne sont pas augmentées avec cette $\operatorname{loi}^{12}$, mais elles conservent de plus leurs avantages au nom de leur "fonction sociale» pourtant fortement contestée (CEDLA, 2014b).

\section{Conclusion : État et capitalisme sauvage dans la Bolivie d'Evo Morales}

Si les ressources naturelles sont au cœur de la politique du gouvernement d'Evo Morales, elles le sont aussi pour le peuple bolivien où les différents groupes s'affrontent pour la définition de leurs imaginaires et de leurs significations autour du développement, de la citoyenneté et de la Nation (Perreault T. et Valdivia G., 2010). Porté par les bases sociales en lutte pour la reconquête des ressources naturelles au nom d'un «nationalisme populaire » (Perreault T., 2006), que ce soit autour de la coca dans les années 1990, de l'eau (2000 et 2004), du gaz (2003), le gouvernement d'Evo Morales a opté à son arrivée au pouvoir pour un "nationalisme étatique » loin de satisfaire l'ensemble des groupes sociaux. La thématique minière est particulièrement révélatrice de ces tensions entre État et groupes auto-organisés. Elle montre de la même manière que si certains mouvements sociaux ont porté un discours revendicatif et alternatif au modèle néolibéral, leur mode de régulation des ressources naturelles reste plus nuancé.

En août 2016, les coopératives minières protestaient ainsi contre une réforme de la Loi des Coopératives autorisant la syndicalisation des travailleurs en leur sein. La mesure gouvernementale avait comme objectif de combler une lacune au sein des coopératives minières où des travailleurs peuvent être embauchés par les «socios" (membres de la coopérative), sans contrôle légal sur les conditions de travail ni les salaires. Pour les dirigeants des coopératives, cette mesure allait cependant à l'encontre du principe même des coopératives et apportait un coût que beaucoup d'entre elles ne pourraient supporter. Des premières manifestations violentes éclatent le 20 août, se soldant par l'arrestation de dix coopérativistes. Le gouvernement accuse le secteur coopérativiste de vouloir profiter du thème de la syndicalisation pour revenir sur l'article 151 de la Loi minière afin d'autoriser les coopératives à souscrire des contrats avec les entreprises privées. Un autre élément du débat était le droit d'utiliser la dynamite lors des mobilisations, instrument « historique » des mineurs, interdit en 2012 puis re-autorisé le $1^{\text {er }}$ mai 2016 sous la pression de la COB. Face à l'échec de la négociation, le vice- 
ministre du Régime intérieur et policier Rodolfo Illanes est envoyé sur place pour négocier. A la suite de l'annonce de plusieurs morts côté coopérativistes lors d'affrontements avec la police, il est finalement séquestré par ceux-ci, torturé puis assassiné.

Suite à cette violence, dans toute la Bolivie de nombreuses voix se sont élevées pour dénoncer les agissements et les manipulations des dirigeants coopérativistes, dont plusieurs ont fait fortune dans l'administration des coopératives, dans la politique ou par la négociation de contrats, éloignés des réalités du travail souterrain. Le gouvernement lança quant à lui une série de décrets: interdiction de l'utilisation d'explosifs lors des manifestations (DS 2888), contrôle fiscal des coopératives (DS 2889), reconversion à l'État des coopératives non productives (DS 2890), reconversion à l' État des coopératives ayant passé des contrats avec des entreprises privées (DS 2891), droit de syndicalisation des travailleurs au sein des coopératives (DS 2892). C'est ainsi une nouvelle « nationalisation » qui est déclarée, non plus à l'encontre des multinationales mais au contraire de l'un des alliés les plus importants du gouvernement.

Cet évènement confirme une nouvelle fois la dépendance de la politique minière $d u$ gouvernement Morales envers ses alliés politiques. Alors que la FSTMB reste ancrée dans une lutte capitaliste traditionnelle contre un patron - qu'il soit public ou privé où l'État reste le principal interlocuteur, les coopérativistes semblent davantage avoir profité de l'État pour promouvoir un capitalisme sauvage à travers l'adoption du modèle néolibéral de refus de régulation étatique et de négociation directe avec les entreprises multinationales. C'est ainsi la dépendance même de l'État à la structuration du marché national et international que révèle cette thématique minière, le gouvernement Morales s'étant montré incapable de transformer l'imaginaire populaire d'un bien commun minier en un bien public au bénéfice de tous.

\section{BIBLIOGRAPHIE}

Arze Alegría, Alejandro, « La lógica del conflicto minero ", in UNIR, La veta del conflicto. Ocho miradas sobre conflictividad minera en Bolivia (2010-2014), La Paz, UNIR, 2014, p.53-80.

Bebbington, Anthony et Humphreys Bebbington, Denise, « Actores y ambientalismos: conflictos socio-ambientales en Perú », Íconos. Revista de Ciencias Sociales, n³5, 2009, p. 117-128.

Crabtree, John et Chaplin, Ann, Bolivia : procesos de cambio, La Paz, PIEB, CEDLA, Oxfam, 2013.

CEDIB, « Porque se debe rechazar todo el proyecto de Ley Minera », CEDIB, 2014, http://

www.cedib.org/wp-content/uploads/2014/04/Analisis-Ley-Minera-1.pdf.

CEDLA, « ¿Obreros o cooperativistas? », Alerta Laboral, n74, 2014a, p.6-7.

CEDLA, « Ley Minera del MAS. Privatista y anti-indigena », Control Ciudadano, n²4, 2014b, p.1-12.

ESTADO PLURINACIONAL DE BOLIVIA, Nueva Constitución Política del Estado, La Paz, Estado

Plurinacional de Bolivia, 2009. 
Francescone, Kirsten et Diaz, Vladimir, « Cooperativas Mineras. Entre socios, patrones y peones », in Petropress, n³0, 2013, p.34-41.

Gudynas, Eduardo, « Diez tesis urgentes sobre el nuevo extractivismo. Contextos y demandas bajo el progresismo sudamericano actual », in CAAP, CLAES (eds), Extractivismo, Politico y Sociedad, Quito, CAAP, CLAES, 2009, p.187-225.

Lacroix, Laurent, «Bolivie : la consultation en débat », séminaire du LAIOS animé par l'équipe SOGIP, Perspectives comparatives sur les droits des peuples autochtones, Paris, 12 décembre 2013.

Le Gouill, Claude, « Imaginaires miniers et conflits sociaux en Bolivie. Une approche multiniveaux du conflit de Mallku Khota », Cahiers des Amériques Latines, Dossier "Greffe ou rejet: l'extraction minière en Amérique latine", № 82, 2016, p.49-69.

Madrid Lara, Emilio, « Conflictos socioambientales en la minería: límites y realidades de su gestión », in UNIR, La veta del conflicto. Ocho miradas sobre conflictividad minera en Bolivia (2010-2014), La Paz, UNIR, 2014, p. 157-172.

Michard, Jocelyn, Cooperativas mineras en Bolivia. Formas de organización, producción y comercialización, Cochabamba, CEDIB, 2008.

Nueva Constitución Política del Estado (NCPE), Bolivia, 2009.

Oporto, Henry, « $i$ La nacionalizacion minera ? Entre el mito y el desencanto », in Oporto, Henry ; Espinoza, Jorge ; Ferrufino, Rubén ; Garzon, Dionisio et Cordova, Hector, ¿De vuelta al Estado minero?, La Paz, Fundación Vicente Pazos Kanki, Foro Minero, 2013, p. 9-47.

Perreault, Thomas, «From the Guerra Del Agua to the Guerra Del Gas: Resource Governance, Neoliberalism and Popular Protest in Bolivia », Antipode, n³8, 2006, p.150-172.

Perreault, Thomas, et Valdivia, Gabriela, « Hydrocarbons, popular protest and national imaginaries: Ecuador and Bolivia in comparative context », Geoforum, vol.41, n5, 2010, p. 689-699.

Perrier Bruslé, Laetitia, « Le gaz bolivien. La Bolivie face à son avenir », Outre-Terre, $\mathrm{n}^{\circ} 18,2007, \mathrm{p}$. 235-251.

Portugal, Pedro, « Visión posmoderna y visión andina del desarrollo », in Wanderley, Fernanda (coord.), El desarrollo en cuestión : reflexiones desde América Latina, La Paz, CIDES-UMSA, Oxfam, 2011, p.253-282.

Poupeau, Franck, «La Bolivie entre Pachamama et modèle extractiviste », Écologie et Politique, $\mathrm{n}^{\circ} 46,2013$, p. 109-119.

Poveda Ávila, Pablo, « Áreas de explotación minera y conflictividad », in UNIR, La veta del conflicto. Ocho miradas sobre conflictividad minera en Bolivia (2010-2014), La Paz, UNIR, 2014, p103-122.

Quiroga Trigo, Maria Soledad, « El sector minero en la perspectiva histórica : trayectoria del actor sociopolítico ", in UNIR, La veta del conflicto. Ocho miradas sobre conflictividad minera en Bolivia (2010-2014), La Paz, UNIR, 2014, p.11-32.

Rodriguez-Carmora, Antonio; Castro, Miguel et Sanchez, Pablo, Imaginarios a cielo abierto. Una mirada alternativa a los conflictos mineros en Peru y Bolivia, Madrid, ACSUR, 2013.

Sandi Bernal, Eliodoro « Minería, desarrollo y conflictividad social », in UNIR, La veta del conflicto. Ocho miradas sobre conflictividad minera en Bolivia (2010-2014), La Paz, UNIR, 2014, p.33-52.

Stefanoni, Pablo et Do Alto, Hervé, Evo Morales, de la coca al palacio. Una oportunidad para la izquierda indígena, La Paz, Malatesta, 2006. 
Svampa, Maristella, « Bolivia. Entre el despliegue de la matriz comunitaria y la actualizacion de lo nacional-popular », in Polet, François (coord), ¿La Bolivia de Evo. Democratica, indigenista y socialista?, Madrid, Editorial Popular, 2010, p. 55-77.

Svampa, Maristella, « Néo-développementalisme extractiviste, gouvernements et mouvements sociaux en Amérique Latine », Problèmes d'Amérique Latine, n81, 2011, p.101-127.

Vanhust, Julia et Beling, Adrian, « Buen Vivir et développement durable : rupture ou continuité ? ", Écologie et Politique, n 46, 2013, p.41-54.

Warhust, Alyson et Bridge, Gavin, « Economic liberalisation, innovation, and technology transfer: opportunities for cleaner production in the minerals industry ", Natural Resources Forum, vol.21, 1997, p.1-12.

Yakovleva, Natalia. Corporate social responsibility in the mining industries, Farnham, Ashgate, Corporate Social Responsibility Series, 2005.

Zegada, Maria Teresa ; Arze, Claudia ; Canedo, Gabriela ; Quispe, Alber, La democracia desde los márgenes : transformaciones en el campo político boliviano, La Paz, CLACSO, Muela del Diablo, 2011.

\section{NOTES}

1. Cette «nationalisation » passe par la refondation de la société publique Yacimentos Petrolíferos Fiscales Bolivianos (YPFB), chargée de gérer et contrôler la commercialisation des hydrocarbures extraits du sous-sol bolivien, et par une renégociation des impôts sur la valeur de la production des hydrocarbures avec les entreprises étrangères, qui se traduit par une hausse du taux d'imposition de 18 à $50 \%$ ( $82 \%$ durant une période de trois mois dite de transition). Cette "nationalisation", accompagnée de la reprise du contrôle d'entreprises par l'État et la renégociation des prix préférentiels du gaz accordés au Brésil et à l'Argentine, a permis de multiplier le budget national par huit entre 2004 et 2012.

2. En Bolivie, c'est l'Impôt direct sur les hydrocarbures (IDH), provenant de la « nationalisation » des hydrocarbures, qui finance une grande partie des politiques sociales.

3. Cette réflexion s'inscrit dans un projet en cours avec l'Observatoire Hommes-Milieux International (OHMI) Pima County (CNRS/Université d'Arizona) sur les formes d'autoorganisation des « communs ».

4. Ce travail est issu de plusieurs années de terrain dans la région minière du Nord Potosí (en doctorat avec le Centre de Recherche et de Documentation des Amériques CREDA) ainsi que d'une série d'entretiens réalisés en avril 2014 avec plusieurs acteurs $\mathrm{du}$ secteur minier bolivien (membres d'organisations sociales, ONG, fonctionnaires, employé du secteur privé) dans le cadre de l'étude « Le processus de changement et la politique extérieure de la Bolivie : risques et perspectives » (CREDA/CNRS), menée avec Laurent Lacroix. Nous axerons notre travail sur les acteurs miniers reconnus par l'État bolivien, en laissant - en partie - de côté les organisations indigènes analysées ailleurs (Le Gouill C., 2016).

5. Etant donné que la rédaction finale de cet article est intervenue quelques jours après cet évènement, il est cependant difficile de dresser un bilan définitif de cet assassinat, le gouvernement d'Evo Morales venant de lancer d'importantes mesures encore difficilement interprétables (voir infra).

6. Ancien président de la COMIBOL (2011-2012) et vice-ministre des mines et de la métallurgie (2010-2011). 
7. Cette industrialisation repose principalement sur le fer du Mutun, des fonderies de zinc à Potosí et Oruro, le projet de raffinerie de Karachipampa et le projet d'industrialisation du lithium du Salar de Uyuni, considéré comme le nouvel « eldorado » bolivien.

8. Celui-ci avait déjà connu une résurgence lors de la mobilisation de 2003 autour de la dénommée " guerre du gaz » contre la vente du gaz bolivien ; puis le secteur coopérativiste a joué un rôle clé dans l'accès au pouvoir d'Evo Morales et la consolidation de sa politique (voir infra).

9. Ces attaques - souvent violentes - sont l'un des problèmes majeurs du secteur minier bolivien (Arze Alegria A., 2014). Certains chiffres estiment qu'il y aurait eu 200 prises illégales de gisement entre 2004 et 2012 ( 45 attaques en 2010 et 58 en 2011). Ces attaques seraient menées par les communautés paysannes-indigènes $(50 \%)$, les coopératives $(30 \%)$ et les travailleurs contre les entreprises privées (20\%) (Portugal P., 2012). D'autres sources estiment cependant que ces communautés ne sont à l'origine que de $12 \%$ des invasions d'aires de travail, alors que le secteur syndicaliste serait à l'origine de 35\% des mobilisations et les coopératives de 28\% (Arze Alegría A., 2014).

10. À Huanuni par exemple, alors que le coût du travail représentait, en $2006,30 \%$ du coût total de la gestion de la mine, il est de 60\% à 70\% en 2012 .

11. Le droit de « consultation préalable peut être défini comme un « instrument de régulation du dialogue, de concertation et de génération d'accords entre peuples autochtones et l'État en ce qui concerne les projets d'exploitation des ressources naturelles dans les territoires autochtones ». D'après les normes internationales, cette consultation doit être « préalable, libre, informée et de bonne foi » (Lacroix, 2013)

12. Une augmentation de $1 \%$ des redevances est cependant légiférée pour l'exploitation de l'or dans les « gisements marginaux ».

\section{RÉSUMÉS}

La Bolivie est un pays de tradition minière où on trouve diverses représentations des ressources du pillage des richesses naturelles par des nations étrangères au rêve "eldoradiste " de développement du pays sur sa base minière. L'arrivée au pouvoir en 2005 du premier président indigène du pays, Evo Morales, devait apporter au pays une nouvelle souveraineté nationale sur ces ressources. Ce modèle s'insère dans l'imaginaire du «Buen Vivir ", qui doit permettre à la Bolivie de dépasser le modèle de développement capitaliste pour proposer un nouveau modèle, plus respectueux de la nature. Malgré de tels objectifs, le gouvernement Morales reste dépendant de l'exploitation des ressources naturelles qui lui permettent de financer ses politiques sociales. Il n'est également pas parvenu à dépasser les conflits entre organisations sociales pour le contrôle des gisements miniers. Tout en qualifiant le secteur minier de «stratégique " pour le développement du pays, le gouvernement Morales définit ainsi davantage sa politique minière pour répondre aux pressions de ces différentes organisations que dans l'intérêt national, comme en témoigne la Loi minière approuvée en 2014. Nous analyserons les contradictions de cette politique à partir de l'étude des différents modes de gouvernances minières en concurrence pour le contrôle des ressources et des réponses politiques apportées par le gouvernement Morales.

Bolivia is historically a mining country where multiple visions concerning the natural resources have emerged, from their plundering by foreign nations to the Eldorado dream of a development of the country on its mining base. The coming to power in 2005 of the first Native American 
president of the country, Evo Morales, was to endow Bolivia with a new national sovereignty on these resources. This project fits into the "Buen Vivir" vision, which is supposed to allow Bolivia to go beyond the model of capitalist development and propose a new model, more respectful of nature. In spite of these objectives, the Morales government remains dependent on the exploitation of natural resources, which allow financing of its social policies. It hasn't succeeded either in transcending conflicts between social organizations for the control of the mining fields. While describing the mining sector as "strategic" for the development of the country, the Morales government designs its mining policy more in answer to pressures from these various organizations than in the national interest, as shown by the Mining Act passed in 2014. I will provide an analysis of the contradictions of this policy, based on the study of the various modes of mining governance in competition for the control of the resources and the political response brought out by the Morales government.

Bolivia es un país históricamente minero en el cual se crearon varios imaginarios, ya sea alrededor del saqueo de sus recursos naturales por una nación extrajera o un sueño « eldoradista » de desarrollo del país a partir de su base minera. La llegada al poder en 2005 del primer presidente indígena, Evo Morales, debía traer al país una soberanía nacional sobre sus recursos. Este proyecto se inserta en el imaginario del "Buen Vivir" el cual debe permitir a Bolivia superar el modelo de desarrollo capitalista proponiendo un nuevo modelo, más respetuoso de la naturaleza. A pesar de estos objetivos, el gobierno de Morales se queda dependiente de la explotación de los recursos naturales que le permite financiar sus políticas sociales. Además, no logró superar los conflictos entre organizaciones sociales para el control de los yacimientos mineros. En el mismo tiempo que describe el sector minero de "estratégico" para el desarrollo del país, el gobierno Morales define más su política minera en respuesta a las presiones de estas diferentes organizaciones que tomando en cuenta el interés nacional, como lo demuestra la nueva Ley Minera aprobada en 2014. Analizaremos las contradicciones de esta política a partir del estudio de los diferentes modos de gobernanza minera en competencia por el control de los recursos y de las respuestas prestadas por el gobierno Morales.

\section{INDEX}

Palabras claves : Bolivia, política minera, Morales, nacionalización, conflicto

Mots-clés : Bolivie, politique minière, Morales, nationalisation, conflit

Keywords : Bolivia, mining policy, Morales, nationalization, conflict

\section{AUTEUR}

\section{CLAUDE LE GOUILL}

Claude Le Gouill est docteur en sociologie de l'Institut des hautes études de l'Amérique Latine (IHEAL) et chercheur associé au Centre de recherche et de documentation des Amériques (CREDA). Il travaille sur les communautés indigènes et paysannes de Bolivie et en particulier sue les questions de développement. claudelegouill@yahoo.fr 